\title{
Magnetohydrodynamic implosion symmetry and suppression of Richtmyer-Meshkov instability in an octahedrally symmetric field
}

\author{
W. Mostert and D. I. Pullin \\ Graduate Aerospace Laboratories, MC105-50, California Institute of Technology, \\ Pasadena, California 91125, USA
}

V. Wheatley

School of Mechanical and Mining Engineering, University of Queensland, Queensland 4072, Australia

R. Samtaney

Mechanical Engineering, King Abdullah University of Science and Technology, Thuwal, Saudi Arabia

(Received 18 May 2016; published 26 January 2017)

\begin{abstract}
We present numerical simulations of ideal magnetohydrodynamics showing suppression of the Richtmyer-Meshkov instability in spherical implosions in the presence of an octahedrally symmetric magnetic field. This field configuration is of interest owing to its high degree of spherical symmetry in comparison with previously considered dihedrally symmetric fields. The simulations indicate that the octahedral field suppresses the instability comparably to the other previously considered candidate fields for light-heavy interface accelerations while retaining a highly symmetric underlying flow even at high field strengths. With this field, there is a reduction in the root-mean-square perturbation amplitude of up to approximately 50\% at representative time under the strongest field tested while maintaining a homogeneous suppression pattern compared to the other candidate fields.
\end{abstract}

DOI: 10.1103/PhysRevFluids.2.013701

\section{INTRODUCTION}

In converging flows, such as that seen in inertial confinement fusion (ICF) [1] and Z-pinch experiments, the presence of Richtmyer-Meshkov (RM) [2-4] and Rayleigh-Taylor (RT) [5,6] instabilities is known to limit the potential for fusion [1]. In flows such as these, the fluids involved are often ionized, and may thus be affected by the application of external magnetic fields. The application of such fields to flows of this kind has been seen in so-called magnetoinertial fusion techniques, from which the magnetized liner inertial fusion (MagLIF) concept has arisen (see Sefkow et al. [7] and references); external fields have been used in the context of electron confinement to field lines with cited increased neutron yields at the hot spot [8,9], and of decreased alpha particle mobility [10]. The effects of such fields on hydrodynamic instabilities in conducting fluids, under the framework of magnetohydrodynamics (MHD), have also been investigated extensively in planar geometries in computational [11,12] and theoretical [13-15] contexts, with the strong suggestion that the RM instability is suppressed due to baroclinic vorticity transport away from the shocked interface by MHD waves. (Slightly different problem formulations, for example where the field is perturbed similarly to the density interface, result in different suppression mechanisms [16].) The motion and effects on the symmetry of the large-scale dynamics in converging MHD flows have also been considered under particular field configurations [17,18], and the RM instability in converging geometry has also been examined in linear [19] and nonlinear [20] computational studies. All of the studies cited here suggest that application of a magnetic field to a flow such as ICF or MagLIF may improve the performance (as defined and measured by respective metrics) of the given technique.

Other methods exist for potentially decreasing the growth of perturbation amplitudes in the converging flow. In ICF these may include doping of the shell material to control density ratios, control of the timing of the laser firing procedure (for instance choosing so-called low-foot and high-foot timing configurations), and ensuring a maximally smooth target shell to mitigate as much 
as possible the seeding of hydrodynamic instability. These and other techniques are reviewed in detail in the context of the National Ignition Campaign by Lindl et al. [1] and are under continuing research. In the effort to reduce perturbation amplitude growth, such techniques would work in complement with judiciously applied magnetic fields as discussed above.

Some of the literature on the dynamics of magnetized converging flows in MHD suggests that the asymmetry of the large-scale dynamics tends to be minimized if the field itself has an increased number of planes of (finite) symmetry [18]. However, the extent of RM or RT suppression may be affected by the use of such a field, particularly in three dimensions, depending on the particulars of the field configuration [20]. In this study we propose a field with octahedral symmetry in order to examine its effect on the RM and to a lesser extent RT instabilities, and the symmetry of the base flows of these problems. Specifically, we compare a magnetic field with octahedral symmetry of order 48 (that is, with 48 spherical symmetry operations including rotations and reflections) with the three-dimensional fields previously considered in Mostert et al. [20], which have dihedral symmetry with order 8 . The study begins with a comparison of the base flow symmetry between these fields, using the primary compressive shock distortion as an indicator, and goes on to interpret and discuss the directional dependence of RM suppression to local field orientation and strength, and the effect of the field configuration on global amplitude growth characteristics. Only spherical flows are considered.

\section{FORMULATION}

\section{A. Equations of motion}

The theoretical framework for this study is ideal MHD with dimensionless variables defined as follows:

$$
\begin{aligned}
& \mathbf{x}=\frac{\hat{\mathbf{x}}}{L_{0}}, \quad t=\frac{\hat{t}}{L_{0} / \sqrt{\hat{p_{0} / \hat{\rho}_{0}}}}, \\
& \rho=\frac{\hat{\rho}}{\hat{\rho_{0}}}, \quad p=\frac{\hat{p}}{\hat{p}_{0}}, \\
& \mathbf{u}=\frac{\hat{\mathbf{u}}}{\sqrt{\hat{p}_{0} / \hat{\rho}_{0}}}, \quad \mathbf{B}=\frac{\hat{\mathbf{B}}}{\sqrt{\mu_{0} \hat{p}_{0}}},
\end{aligned}
$$

where $\rho, \mathbf{v}, \mathbf{B}$, and $p$ are the density, velocity vector, magnetic field vector, and pressure, respectively, $\mu_{0}$ is the free space permeability, $L_{0}$ defines the (dimensional) length scale, and carets otherwise indicate dimensional variables. In the absence of a gravitational potential, the ideal MHD equations may be written [21]

$$
\begin{gathered}
\frac{\partial \rho}{\partial t}+\nabla \cdot(\rho \mathbf{v})=0 \\
\rho\left(\frac{\partial \mathbf{v}}{\partial t}+\mathbf{v} \cdot \nabla \mathbf{v}\right)+\nabla p-(\nabla \times \mathbf{B}) \times \mathbf{B}=0 \\
\frac{\partial e}{\partial t}+\mathbf{v} \cdot \nabla e+(\gamma-1) e \boldsymbol{\nabla} \cdot \mathbf{v}=0 \\
\frac{\partial \mathbf{B}}{\partial t}-\nabla \times(\mathbf{v} \times \mathbf{B})=0
\end{gathered}
$$

with $e$ the specific internal energy, related to the pressure by

$$
e=\frac{p}{(\gamma-1) \rho}
$$


and $\gamma$ the specific heat ratio, which for an assumed thermodynamically perfect, monatomic gas is $\gamma=5 / 3$. Note that the magnetic field is also solenoidal. In ideal MHD, diffusive effects are assumed to occur over a sufficiently larger time scale than advection effects, and are thus neglected.

\section{B. Problem geometry and interface initialization}

We define a Cartesian, three-dimensional geometry with coordinates $(x, y, z)$. For descriptive purposes and to aid in some aspects of the initialization, spherical coordinates $(r, \phi, \theta)$, with $\theta$ the polar angle (colatitude), are also used as defined in the usual manner. A Riemann problem is initialized by a spherical Riemann interface (RI), centered at the origin with radius $R_{R}$, separating two quiescent fluids which differ in density and pressure by a factor of 3 and 12.1, respectively, where the low-density, low-pressure fluid is on the inside of the interface. This particular formulation allows the formation of inward-traveling fast and slow MHD shocks, of which the fast shock is of particular interest for its symmetry characteristics, and two outward-traveling MHD rarefaction waves, whose presence is incidental. The chosen density and pressure ratio over the RI allows an initial sonic Mach number of 2 for the fast MHD shock, on the $x$ axis. This initial Mach number is not uniform over the interface since the geometry of the magnetic field, discussed in Sec. II C, in general affects the speed of the fast shock generated by the Riemann problem depending on the local field orientation and strength.

Flows both with and without a density interface (DI) in addition to the RI are considered. In the absence of a DI, which is used for the RM problems and defined below, the Riemann problem is used to examine the effect of the magnetic field on the large-scale asymmetry of the base flow. In the presence of a DI, the Riemann problem is used to accelerate the DI to provoke the RM instability. Defining now this DI, we consider single-interface flows as follows. A perturbed DI is placed at a radius $R_{0}<R_{R}$ (that is, on the inside of the $\mathrm{RI}$ ). The fast shocks generated by the Riemann problem will accelerate the DI, provoking the RM instability. We define the DI by a spherical harmonic formulation similar to that of Lombardini et al. [22] with the surface $\zeta_{0}(\theta, \phi)$ according to the expansion

$$
\zeta_{0}(\theta, \phi)=r_{0}-\eta_{0} \sum_{l=0,2,4, \ldots}^{\infty} \sum_{m=0,2,4, \ldots}^{l} f_{l m} Y_{l m}(\theta, \phi),
$$

with coefficients

$$
f_{l m}=\sqrt{(2 l+1) C_{l}} \frac{\cos \left(2 \pi \omega_{l}^{m}\right)}{\sqrt{\sum_{j=-l}^{l} \cos \left(2 \pi \omega_{l}^{j}\right)^{2}}},
$$

where $\omega_{l}^{m}$ and $\omega_{l}^{j}$ are randomly generated numbers in $[0,1]$ and $C_{l}$ is an "angular power spectrum"

$$
C_{l}=\frac{1}{4(2 k+1)} \frac{1}{\sigma_{0} \sqrt{2 \pi}} \exp \left(-\frac{(l-k)^{2}}{2 \sigma_{0}^{2}}\right) .
$$

The quantity $k$ is a "dominant spherical wave number," which we set $k=32$, and $\sigma_{0}$ is a standard deviation, set $\sigma=1$. Equation (7) differs from that of Lombardini et al. [22] and Mostert et al. [20] by selecting only even $l$ and $m$, with $m \geqslant 0$. This ensures that the density interface is normal to the boundaries at $\phi=0, \pi / 2$ and $\theta=0, \pi / 2$, so that the principal planes $x, y, z$ are true symmetry planes for the initial density interface. The amplitude is set such that the standard deviation of the interface position is approximately $4 \%$ of the wavelength. The DI and RI are regularized with a hyperbolic tangent profile with $\mu=270, \mu$ being the tanh frequency, following Mostert et al. [20]. In this study only light-heavy interface flows with $\mathcal{A}=2 / 3$ are considered, where $\mathcal{A}$ is the Atwood number defined

$$
\mathcal{A}=\frac{\rho_{\text {in }}-\rho_{\text {out }}}{\rho_{\text {in }}+\rho_{\text {out }}}
$$

with $\rho_{\text {in }}$ and $\rho_{\text {out }}$ the inner and outer densities, respectively. 


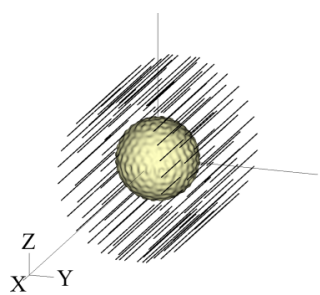

(a)

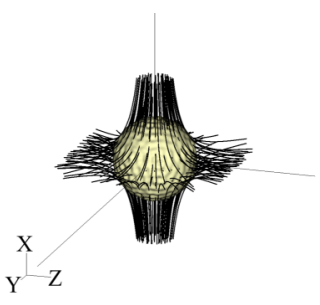

(b)

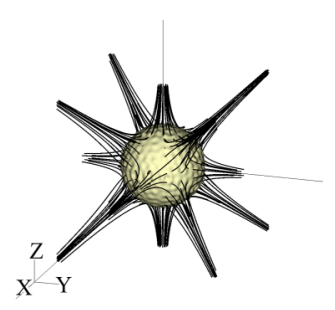

(c)

FIG. 1. Initial conditions showing (single) density interface (DI) (beige) and magnetic field lines (black) in three dimensions for the various field configurations. Simulation is done on the octant domain. For clarity, the two-loop field is shown with the $x$ axis pointing up. (a) Uniform field, (b) Two-loop field, and (c) Octahedral field.

\section{Magnetic field configurations}

The magnetic fields used in this study can be seen in Fig. 1 around an example density interface. The uniform and two-loop fields have been previously considered by Mostert et al. [20] and are used again here for comparison with the presently considered third field, which is octahedrally symmetric. The reference magnetic field strength $B_{0}$ is set according to the field strength parameter, defined as the ratio of thermodynamic to magnetic reference pressures,

$$
\beta_{0 I}=\frac{2 p_{0}}{B_{0}^{2}} .
$$

The reference field strength is taken on the DI, on the $x$ axis in each case. The field configurations are formulated as follows:

Uniform, unidirectional field.

$$
\mathbf{B}=B_{0} \hat{\mathbf{e}}_{\mathbf{x}}
$$

following Mostert et al. [20], and where $\hat{\mathbf{e}}_{\mathbf{x}}$ is the $x$-unit vector. This field may be physically realized by the placement of a current coil across the domain, oriented in the $x$ direction. This field is considered dihedrally symmetric with a symmetry order of 8 . This means that in a spherical domain, the smallest volume containing the field that could be used to represent the field in the whole through a combination of translations and reflections has a volume an eighth of the domain.

Two-loop field. This field results physically from the placement of a pair of current loops with radius $R_{L}$ at Cartesian coordinates $\left(q_{i}, 0,0\right)=\left( \pm X_{0}, 0,0\right)$ (where $q_{i}$ is the location of the $i$ th current loop). Each current loop is oriented normally on its respective axis. The magnetic field at a given point in space is then calculated according to Smythe [23]:

$$
\begin{aligned}
& B_{a x, i}=\alpha_{i} B_{0} \frac{1}{\pi \sqrt{Q_{i}}}\left[E\left(\kappa_{i}\right) \frac{1-\varrho^{2}-\chi_{i}^{2}}{Q-4 \varrho}+K\left(\kappa_{i}\right)\right], \\
& B_{r a, i}=\alpha_{i} B_{0} \frac{\zeta_{i}}{\pi \sqrt{Q_{i}}}\left[E\left(\kappa_{i}\right) \frac{1+\varrho^{2}+\chi_{i}^{2}}{Q-4 \varrho}-K\left(\kappa_{i}\right)\right],
\end{aligned}
$$

following Mostert et al. [20], where the subscripts $a x$ and $r a$ signify components in the axial and plane-radial directions of the $i$ th current loop, respectively. The total magnetic field is calculated from the summed contributions from each current loop using (13) and (14). $Q_{i}=(1+\varrho)^{2}, \kappa_{i}=\sqrt{4 \varrho / Q_{i}}$, $\varrho=r_{r a} / R_{L}, \xi_{i}=\left(q-q_{i}\right) / r_{r a}$, and $q-q_{i}$ is the axial distance from the current loop. For example, if a current loop is centered on the $x$ axis (as is the case for this field configuration), then $q=x$. $E$ and $K$ are respective elliptic integrals of the first and second kind (calculated here according to the algorithm of Carlson [24]), and $r_{r a}$ is the projected radial position for the $i$ th current loop. $\alpha_{i}=\left\{\alpha_{0},-\alpha_{0}\right\}$ scales the magnetic field such that the magnetic field strength parameter $\beta_{0 I}=2 p_{0} /\left|\mathbf{B}\left(R_{0}\right)\right|^{2}$, and 


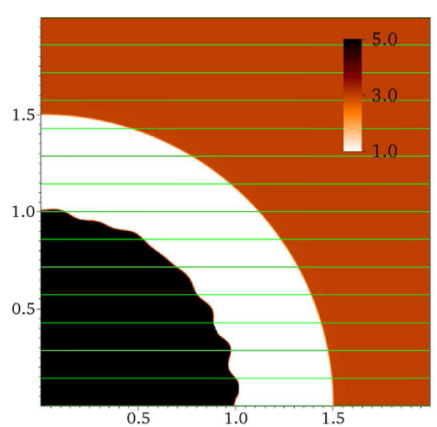

(a)

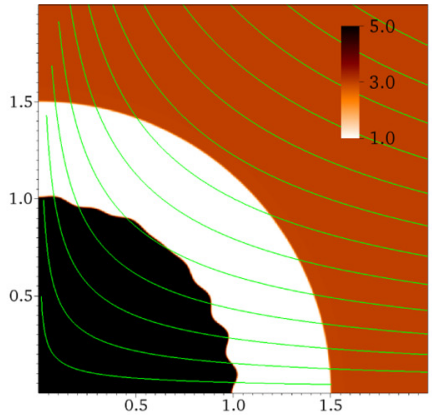

(b)

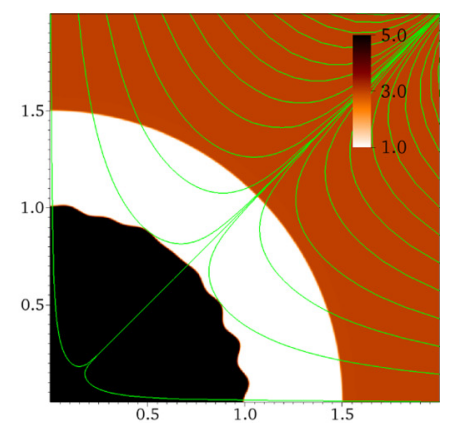

(c)

FIG. 2. Initial conditions, shown in $x-y$ plane, for cases (a) L1, (b) L2, and (c) L6 (see Table I for description of case abbreviations). The outer interface (Riemann problem interface) generates the shocks to accelerate the perturbed density interface (inner). Magnetic field lines are overlaid in green.

$N=2$ is the number of current loops. Hence, it is referred to here by the shorthand term "two-loop field." This field can be considered dihedrally symmetric with a symmetry order of 8 . Hence, it has the same symmetry as the uniform-field configuration. However, its two-dimensional analog, considered in Mostert et al. [18,20], has an additional reflection axis over the uniform-field case. Here, in a plane containing the $x$ axis, as in Fig. 2(b) below, the magnetic field resembles its two-dimensional analog and in this sense has greater symmetry over the uniform case.

Octahedrally symmetric field. For this field configuration, three pairs of current loops are placed at $\left( \pm X_{0}, 0,0\right),\left(0, \pm Y_{0}, 0\right),\left(0,0, \pm Z_{0}\right)$, with a magnetic field at a given point in space calculated using (13) and (14) summed over $N=6$ loops with scaling factors $\alpha_{i}=\left\{\alpha_{0},-\alpha_{0}, \alpha_{0},-\alpha_{0}, \alpha_{0},-\alpha_{0}\right\}$. This field has octahedral symmetry with a symmetry order of 48 , and in this sense more closely approximates spherical symmetry than the other two candidates. It is referred to here as the octahedrally symmetric field, or octahedral field, to stress its symmetry properties, or equivalently as the "six-loop field" to refer to its physical arrangement.

\section{Magnetic field strength}

For this study, and in particular for comparisons between the uniform and octahedral cases, we consider three magnetic field strengths, which are chosen $\beta_{0 I}=32,16$, and 8 . They are motivated in part by the field strengths considered in previous studies such as Mostert et al. [20], but the strongest is chosen in particular according to the critical field strength parameter derived by Sano et al. [25] given in that study by

$$
\beta_{\text {crit }} \equiv \frac{2}{\gamma} \alpha^{-2}\left(\frac{c_{s 2}^{*}}{v_{\text {lin }}}\right)^{2}\left(\frac{P^{*}}{p_{0}}\right)^{-1},
$$

where $v_{\text {lin }}$ is the linear, hydrodynamic growth velocity of the shocked interface, $\alpha$ is an adjustment factor accounting for the nonlinear growth phase, usually of order $0.1[26,27], c_{s 2}^{*}$ is the sound speed in the postshock light side of the interface, $P^{*}$ is the postshock pressure on the interface, and $p_{0}$ is a reference pressure as above. Sano et al. note directly that for weak shocks (for example, $M \leqslant 3$ ), fields with $\beta \geqslant 100$ may effectively suppress the RM instability. A conservative estimate in our formulation using (15) suggests $0.5<\beta_{\text {crit }}<20$ depending on the ratio $c_{s 2}^{*} / v_{\text {lin }}$. Note furthermore that Sano et al.'s study regards in particular the RM instability in normal field orientation, for which the RM instability may not be as effectively suppressed as for parallel interface-field orientations [20], although Sano et al. note similar values of $\beta_{\text {crit }}$ in their simulations with parallel orientations [25]. In light of these approximations, we regard the estimate for critical field presented here as a region in which our field is sufficiently strong to suppress the RM instability in the sense that 
TABLE I. Description of case abbreviations accounting for the types of problem, field configuration, and nondimensional strength $\beta_{0 I} . \beta_{0 I}=\infty$ indicates an unmagnetized case (zero field strength).

\begin{tabular}{lllr}
\hline \hline Case abbreviation & \multicolumn{1}{c}{ Type of problem } & \multicolumn{1}{c}{ Field configuration } & $\beta_{0 I}$ \\
\hline L0 & Richtmyer-Meshkov & None (hydrodynamic) & $\infty$ \\
L1-32 & Richtmyer-Meshkov & Uniform unidirectional & 32 \\
L1-16 & Richtmyer-Meshkov & Uniform unidirectional & 16 \\
L1-8 & Richtmyer-Meshkov & Uniform unidirectional & 8 \\
L2-32 & Richtmyer-Meshkov & Two-loop & 32 \\
L6-32 & Richtmyer-Meshkov & Octahedrally symmetric & 32 \\
L6-16 & Richtmyer-Meshkov & Octahedrally symmetric & 16 \\
L6-8 & Richtmyer-Meshkov & Octahedrally symmetric & 8 \\
N1-8 & Pure Riemann problem & Uniform unidirectional & 8 \\
N2-8 & Pure Riemann problem & Two-loop & 8 \\
N6-8 & Pure Riemann problem & Octahedrally symmetric & 8 \\
\hline \hline
\end{tabular}

Sano et al. consider, and we expect the field strengths $\beta_{0 I}=8,16$ to be sufficiently strong for RM suppression.

\section{E. Case abbreviations}

Finally, we designate abbreviations for each problem. An abbreviation is prefixed with " $L$ " for the light-heavy interface problem and "N" for the no-interface problems used to examine symmetry of the base flows, followed by " 0 " for the hydrodynamic (no-field) case, " 1 " for uniform field, " 2 " for the two-loop field, and "6" for the six-loop field, which is octahedrally symmetric. This number signifies the number of current loops that may be used to physically generate the field in question. A suffix is used to signify the field strength, $\beta_{0 I}$. Table I outlines all the problems considered in this study. In addition, though they are not explicitly labeled here, versions of each magnetized RM problem with unperturbed DIs are also run to aid in calculation of the diagnostics of perturbation growth.

Figure 2 shows the initial condition in density, in the $x-y$ plane, for some of the problems listed in Table I. Note that in the $y-z$ plane, not shown in Fig. 2, the field lines are everywhere normal to the plane in L1 and everywhere tangential to the plane in L2 (at $x=0$ ).

\section{METHODOLOGY}

We use a second-order nonlinear compressible upwind finite volume method developed by Samtaney et al. [28], which uses a Roe-type Riemann solver and a projection method to enforce the solenoidal property of the magnetic field. We simulate on a Cartesian adaptively refined octant-domain mesh of the Berger-Colella type, using the Chombo framework [29], with nondimensional extents $(0,2)$ in each principal direction; we use an unrefined mesh size of $64^{3}$ with three levels of refinement for an effective resolution of $512^{3}$, and a refinement criterion based on local density gradient, $|\nabla \rho|>0.02 \rho$. This mesh is sufficiently refined to resolve the maximum-amplitude perturbation according to the study by Mostert et al. [20]. In fact, in that study the physical domain was larger and the initial perturbation amplitude root-mean-square smaller than here, for the same total refined resolution; hence the present effective resolution per wavelength is higher than in the cited study. For the cases at stronger field $\left(\beta_{0 I}=8,16\right)$, we use nondimensional extents $(0,1.7)$ with the same mesh size to avoid very large field strengths at the outer boundaries.

It is possible that the outward-moving waves generated by the Riemann problem may reflect in some manner off the outer boundaries of the domain and potentially interact with the main features of the flow at later times. In the results presented here, although such interactions may occur, they do not present themselves in any meaningful way and they are most likely dominated by the effects of 


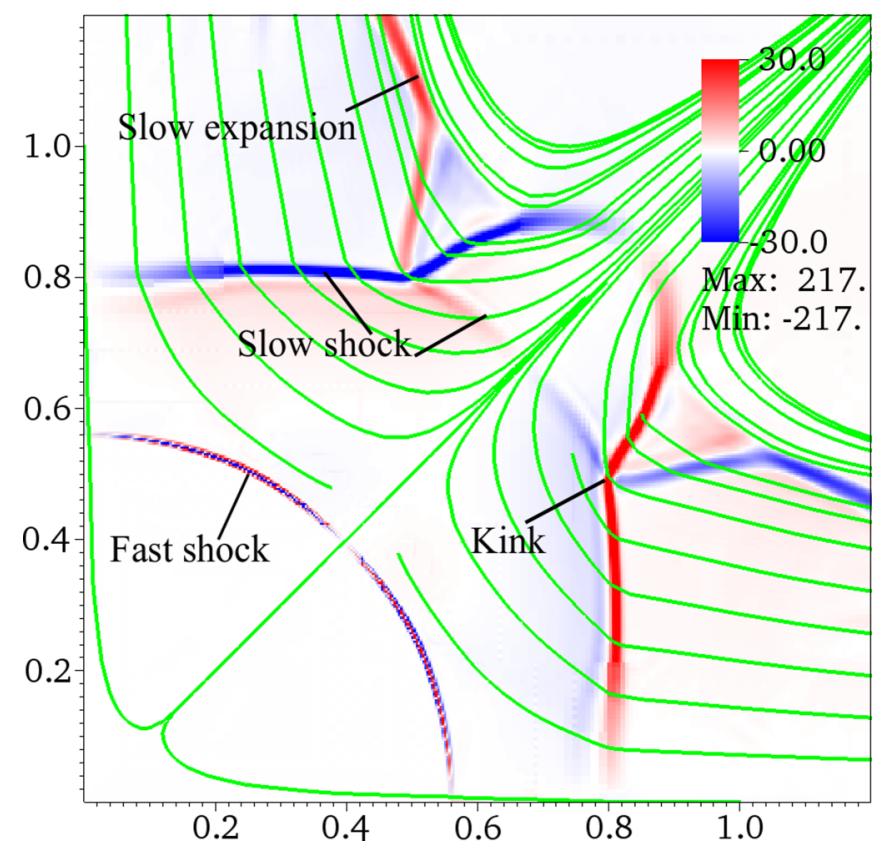

FIG. 3. $z$ vorticity in the $x-y$ plane showing major features of the Riemann problem N6-8 (see Table I for description of abbreviations) at $t=0.32$. Magnetic field lines projected into the $x-y$ plane overlaid in green. Fast shock, slow shock structure, slow expansion, and "kink" described. Fast expansion is not visible.

high pressure and magnetic field gradients near the features of interest. Hence, their potential effects are not considered in this study.

\section{RESULTS}

\section{A. Base flow}

Before considering the RM problems in detail, we first characterize the base flow in the presence of the octahedral field in order to observe the effect of the field on the implosion symmetry. That is, we characterize the spherical Riemann problem in the absence of a DI, described by the case N6-8 in the context of the previously considered cases N1-8, N2-8 (see Table I for description of abbreviations). The N6-8 Riemann problem is shown in Fig. 3 at a representative time. The waves present are, ordered outwards from the center, a fast MHD shock, a slow MHD shock, a contact discontinuity (CD), a slow MHD expansion, and a fast MHD expansion (see for example Fig. 2 of Mostert et al. [20], in which this sequence of waves is also prevalent). Qualitatively, as for the other field configurations [18], the structure of the Riemann problem also exhibits symmetry equivalent to that of the magnetic field, owing to the variation of the magnetic field vector over the domain and hence of the various MHD characteristic speeds. In particular, there are reflections (denoted "kinks" by Mostert et al. [18]) in the slow MHD shock system at values of $\phi, \theta$ where the slow magnetosonic speed is at a local minimum (in fact, locally zero), or equivalently where the magnetic field is locally parallel to the shock surface. These are clearly visible in Fig. 3.

We are interested in how the symmetry of the octahedral-field base flow compares to that of the other, previously considered cases. A key metric for the base flow symmetry is the symmetry of the imploding fast shock system, having been examined previously to find that the uniform field case suffers from a clear asymmetry in its fast shock system particularly at high field strengths [18], while the two-loop field provides a more symmetric implosion (at a slight cost to RM suppression [20]). Here we measure the shock asymmetry by taking the mean and root-mean-square (rms) deviation in 


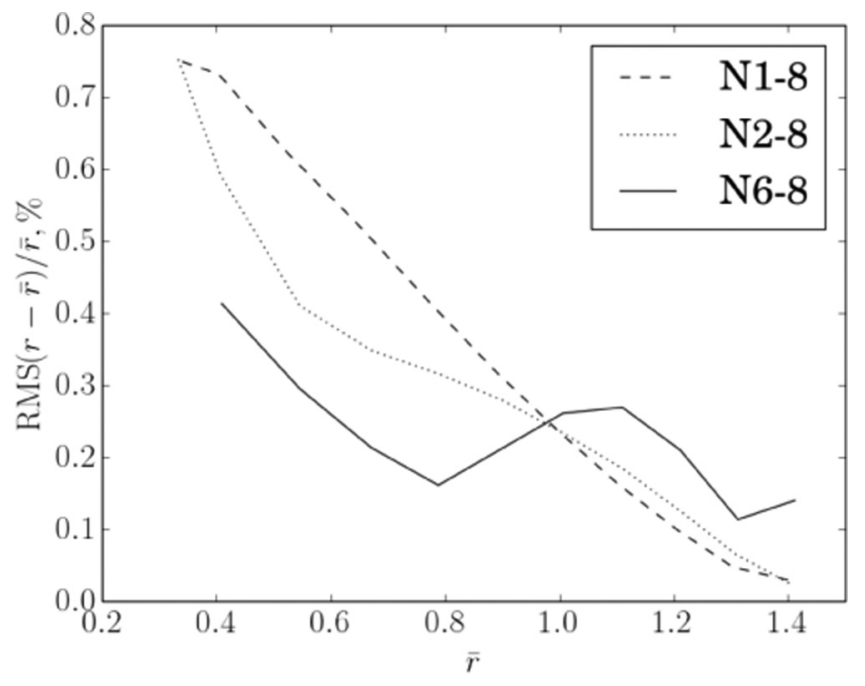

FIG. 4. Asymmetry evolution of the fast shock systems of the cases N1-8, N2-8, and N6-8 as they collapse to the origin in the pure Riemann problem (see Table I for case abbreviations). Abscissa shows mean shock position; ordinate shows root-mean-square deviation in shock position normalized by the mean. The octahedral-field case exhibits a greater degree of symmetry in its fast system over the other cases for all but early times in the collapse. The brief spike in the octahedral case at early time is due to greater influence of the field at larger shock radii.

radial position of the $p / p_{0}=3$ isosurface, taking this isosurface to describe the shock position and using its statistics to describe the fast shock symmetry. Figure 4 compares the octahedral-field base flow N6-8 to the uniform case base flow, showing the variation of rms shock position (normalized by its mean position) as it collapses to the origin. A high rms is considered to indicate a more asymmetric flow.

Immediately from Fig. 4 the octahedral-field case can be seen to show a lesser degree of asymmetry over the other cases for all but early times (large shock radii). While the greater symmetry of the field is a contributor to this, there are present two other very influential factors which stem from a common effect. First, note that the field strength parameter $\beta_{0 I}$ is defined at the radius $r=1$ in anticipation of the placement of the DI there in the RM cases considered below. While in the uniform-field case this is not important since the field is (obviously) uniform everywhere, in the octahedral-field case the field strength increases dramatically with radius. This leads to a more pronounced effect of the field's nonspherical symmetry on the shock system symmetry at startup of the Riemann problem-that is, for $r>1$. The two-loop case (N2-8) does not show as high an asymmetry at early times, probably since the field increase past $r=1$ is not as dramatic as in the octahedral case. Second, the field continues to decrease in strength as the shock collapses through $r<1$ in the octahedral case, further reducing the effect of the field and its asymmetries as the shock collapses.

Thus, there are two primary contributors to the increased symmetry of the octahedral field base flow over the other cases: the increased degree of spherical symmetry of the field, and the decreasing influence of the field on the shock system as it collapses. It is important to note that while the two-loop field has dihedral symmetry similarly to the uniform field case, it does benefit from the second of these contributors, as observed in a previous study [20].

\section{B. RM instability}

\section{Effect of field symmetry}

Equipped with an understanding of the base flow, we proceed now to comparison of the RM cases. In particular, we wish to compare interface perturbation growth of the uniform, two-loop, 


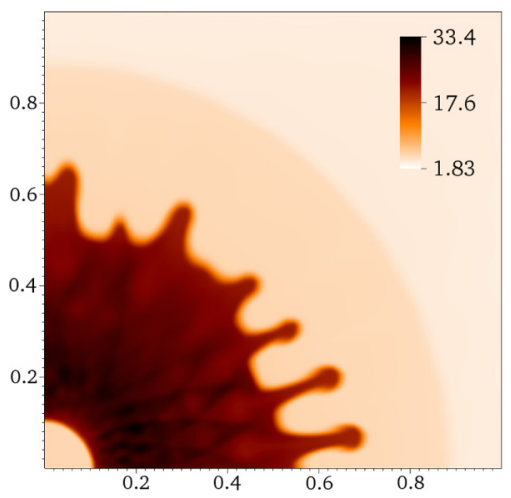

(a)

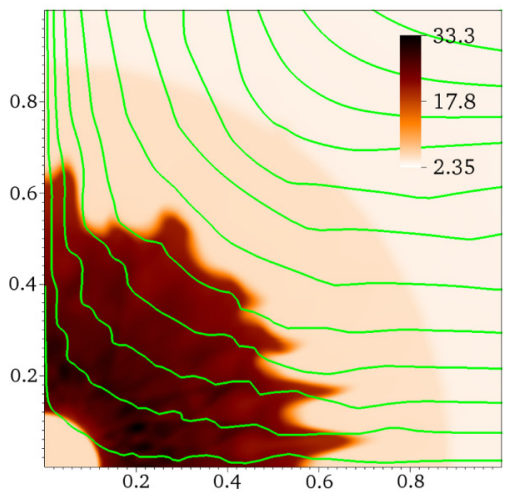

(c)

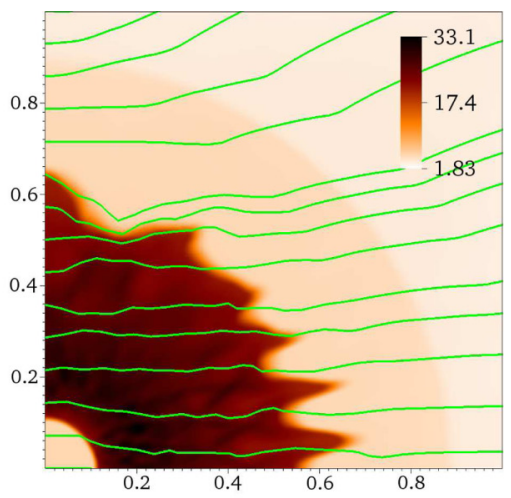

(b)

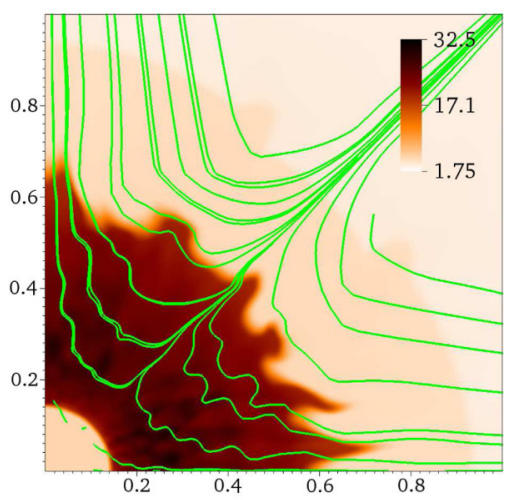

(d)

FIG. 5. Density field in the $x-y$ plane at comparable times for L cases (see Table I for description of abbreviations) with $\beta_{0 I}=32$ showing overlaid magnetic field lines. Growth due to the RM instability is visibly reduced in each magnetized case when compared to the unmagnetized (L0) case. The transmitted fast shock is at approximate nondimensional radius 0.1 in each case. The $x-z$ plane shows a similar density field and plane-projected field lines. (a) $\mathrm{L} 0, t=0.593$, (b) $\mathrm{L} 1-32, t=0.592$, (c) $\mathrm{L} 2-32, t=0.590$, and (d) $\mathrm{L} 6-32, t=0.586$.

and octahedral cases, with the hydrodynamic case as the unmagnetized reference. The uniform and two-loop field configurations were previously considered by Mostert et al. [20] so are not in principle new, although the initial conditions here differ from the previous study. We begin with a comparative study between all cases at the weakest field strength $\beta_{0 I}=32$, followed by a comparison between the uniform and octahedral cases across all strengths. In the cases considered, the primary accelerating shock is the fast MHD shock. The slow MHD shock may also influence the DI, and in strong magnetic fields the slow shock system has been known to severely disrupt the symmetry of the DI [20]. However, this effect appears in only the strongest cases and is not examined in detail here.

Figure 5 shows snapshots of the density field, with magnetic field lines overlaid, at comparable times for L0, L1-32, L2-32, and L6-32 in the $x-y$ plane. The suppression mechanism is the transport of vorticity away from the interface roughly along field lines, as extensively studied previously [11-14,20,30], by sub-fast MHD waves arising from the shock refraction process as the fast shock system processes the DI.

Visualizing the same flows now in vorticity, Fig. 6 shows that vorticity transport from the interface occurs for all magnetized cases as it also shows the relative positions of the fast and slow shock systems. The slow shock system remains distant from the DI for cases L1-32 and L2-32, but for 


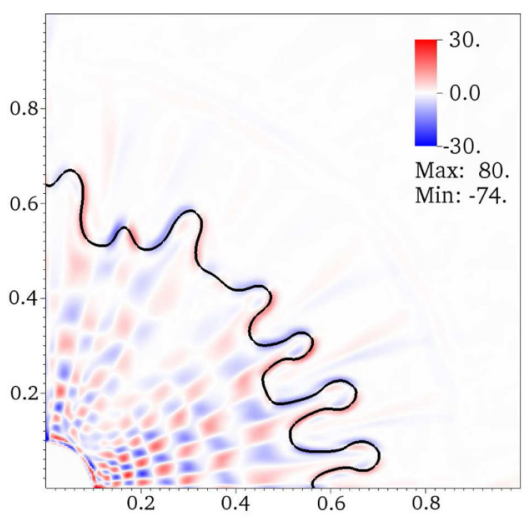

(a)

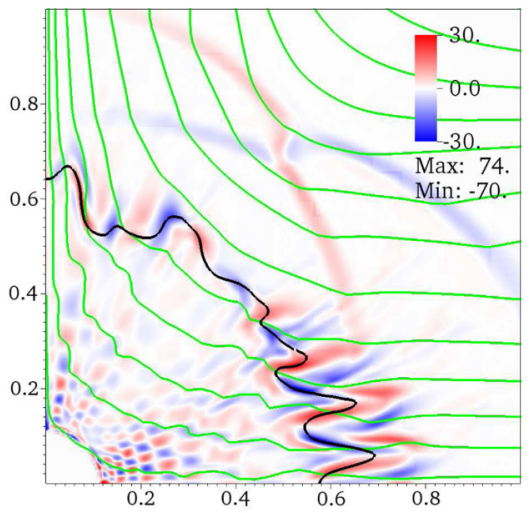

(c)

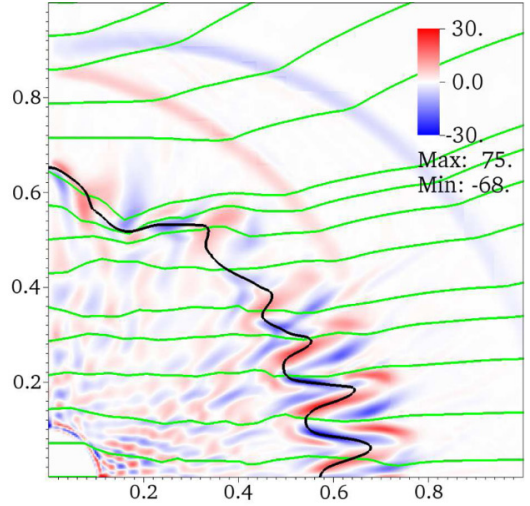

(b)

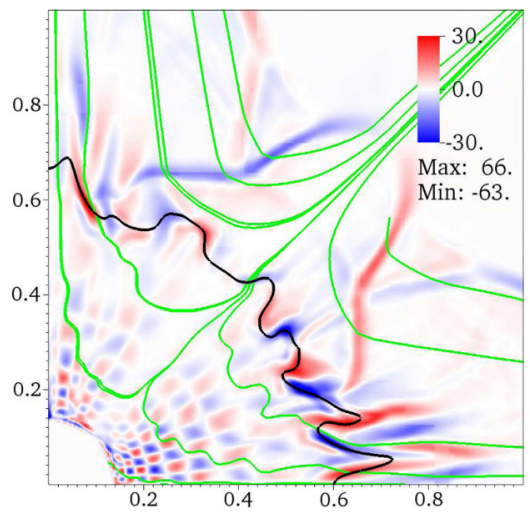

(d)

FIG. 6. $z$ component of vorticity at comparable times for $\mathrm{L}$ cases $\left(\beta_{0 I}=32\right)$ with overlaid magnetic field lines. Interface position shown in black contour. Vorticity remains on the interface for the unmagnetized (L0) case, but is transported roughly along field lines, according to the field geometry, in each magnetized case. Although vorticity remains close to the interface where the field is tangential to it, RM growth is reduced there. The $x-z$ plane shows a similar $y$-vorticity field and plane-projected field lines. (a) $\mathrm{L} 0, t=0.593$, (b) $\mathrm{L} 1, t=0.592$, (c) L2, $t=0.590$, and (d) L6, $t=0.586$.

L6-32 it may influence the DI directly, by refraction through it, or indirectly by disturbing vorticity transport near the DI. It does, however, not appear to affect the global perturbation amplitude growth (see Fig. 7) or the general appearance of the DI.

Figure 7 shows the root-mean-square perturbation amplitude across the domain for each case. The amplitudes for perturbations in cases L1, L2, L6 are estimated by subtracting the interface position from that of the unperturbed simulations. This is done so that large-scale variation of the interface position due to the magnetic field - a large-scale dynamic effect influenced by asymmetry in the fast shock systems (see Sec. IV A) — does not interfere with the estimation of local amplitudes. In each case (barring L0), the interface position vector for the unperturbed case is interpolated onto the coordinates $(\phi, \theta)$ in the perturbed case, which allows a pointwise subtraction of one from the other. Such a subtraction need not be performed in the case L0, since the unperturbed interface in that case is clearly a sphere with radius equal to the mean position of the perturbed interface. Note the amplitude defined in this way is necessarily positive. The growth curve for L6 is comparable to that of L1 in particular, suggesting that the RM instability is suppressed to the same extent, globally, between the uniform unidirectional and the octahedrally symmetric fields. Of the fields examined, 


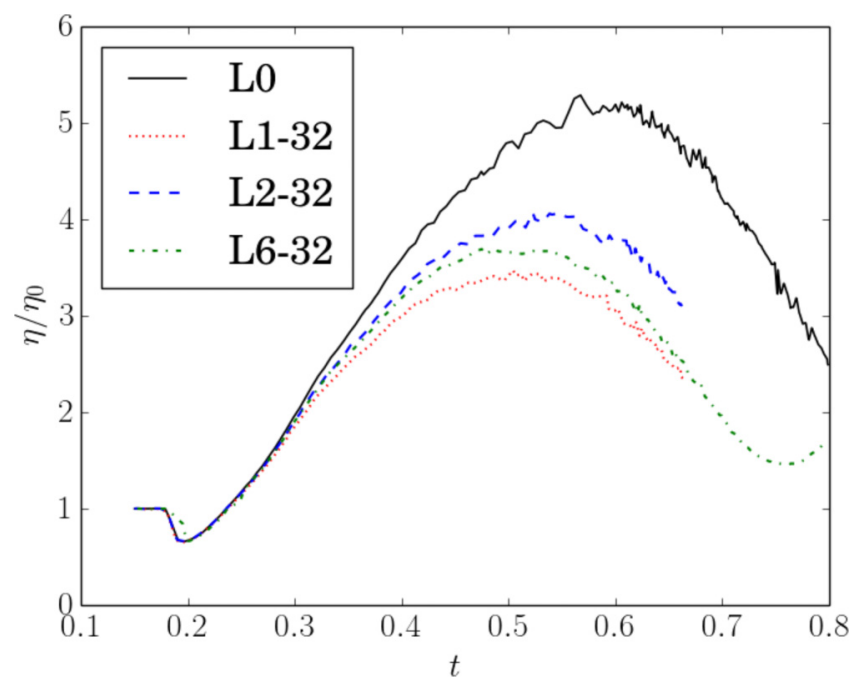

FIG. 7. Root-mean-square perturbation amplitude, taken across entire octant domain, for cases L0, L1, L2, L6 (see Table I). All magnetized cases show reduced RM growth over the unmagnetized (L0) case, with the most reduction seen in case L6.

L2 suppresses the RM instability the least in a global sense. At very late times, the curve for L6-32 can be seen to increase again. This apparent increase is due to the RT-driven phase inversion of the perturbations on the interface, and in fact occurs in all flows with late-time RT effects (see Mostert et al. [20] for further examples).

The transport of vorticity away from the interface is particularly clear on the axes $(\phi=0, \pi / 2)$ in each case since the field is normal to the interface at these $\phi$ for L2-32 and L6-32. In L6-32, while the field is also locally normal to the interface at $\phi=\pi / 4$, the vorticity remains close to the interface even at late times since the field is weak on that part of the interface. The primary difference in RM suppression in this plane between L2-32 and L6-32, then, should be in how the DI evolves around $\phi=\pi / 4$, due to the differing field orientation there. However, the vorticity transport pattern visible in Figs. 5(c) and 5(d) at this angle from the horizontal appears very similar between L2-32 and L6-32. Thus, the differences in RM suppression between these cases must occur in another plane.

Consider then the $y-z$ plane, where the plane-projected field geometries are very different between all cases. Figure 8 shows the $x$ vorticity in this plane for the different field configurations. L1-32 does not show plane-projected field lines since they are everywhere normal to the plane. First, in comparing Figs. 8(b) and 8(c), perturbations appear smaller in case L1-32. The reasons for this are twofold. First, the field magnitude is stronger at all points on the interface in this plane for L1-32 than in L2-32, naturally providing stronger RM suppression. Second, the field is everywhere tangential to the interface in case L1-32, which as mentioned above provides a greater suppressive effect than in L2-32, where the field is everywhere normal in this plane.

The field L6-32 is also non-normal to the interface for most of its extent. Furthermore, the field strength on the interface in this plane is on average stronger than in L2-32. These two factors immediately explain the greater L6-32 RM reduction seen in Fig. 7. In fact, looking at Fig. 9, which shows a probability density function (PDF) of strength across the entire interface for each configuration, we see that the octahedral field in fact tends to be stronger in an overall sense than the two-loop field for this $\beta_{0 I}$, despite its tail to weaker field strengths. This highlights an important feature of the two-loop configuration: among the three Cartesian planes, there is one plane in which it behaves differently with respect to the RM instability from the others, and it happens to be the less effective in RM suppression there. The same is true for the L1 configuration, except that in its odd plane it is slightly more effective in RM suppression than in the other Cartesian planes. Nonetheless, 


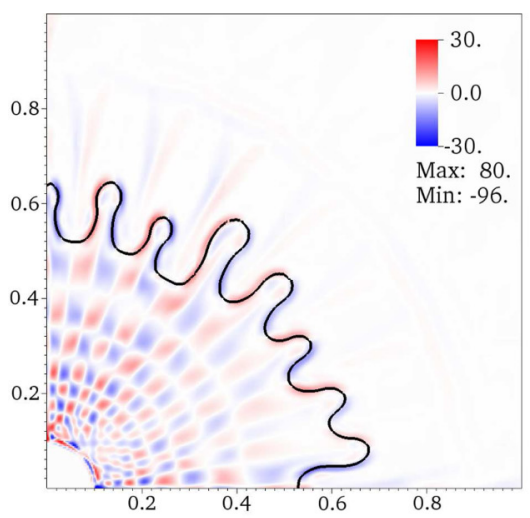

(a)

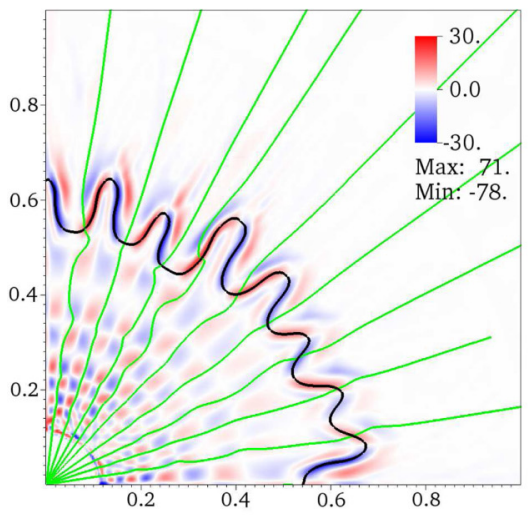

(c)

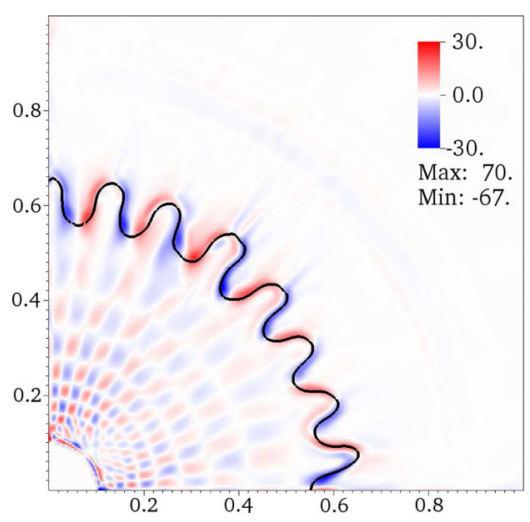

(b)

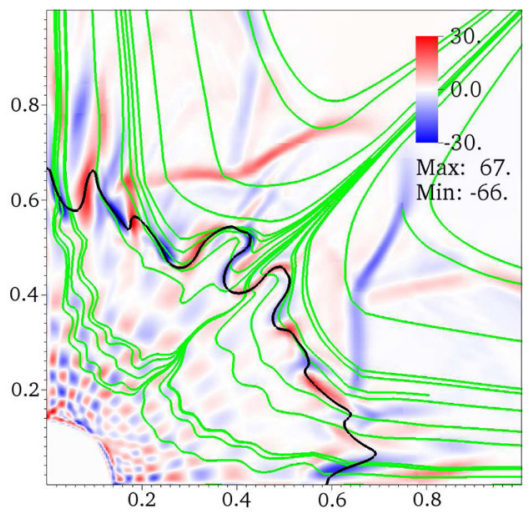

(d)

FIG. 8. $x$ vorticity at comparable times (the same as in Fig. $6, \mathrm{~L}$ cases at $\beta_{0 I}=32$ ), showing now the $y-z$ plane with overlaid magnetic field lines. This plane is distinct from the $x-y$ and $x-z$ planes in particular for the L1 and L2 cases since the plane-projected field is different here. Field lines are not shown for the L1 case since they are everywhere normal to the plane. Note the similarity between Figs. 6(d) and 8(d). (a) L0, $t=0.593$, (b) $\mathrm{L} 1, t=0.592$, (c) $\mathrm{L} 2, t=0.590$, and (d) L6, $t=0.586$.

the uniform field sees a very different expression of the RM instability in this plane as a result of its axisymmetry there. Owing to its symmetry, the octahedral configuration does not suffer from these characteristics, and provides similar effects on the RM instability in mode and extent in each of the Cartesian planes. The effect that this has on the shape of the perturbations on the DI is explored in Sec. IV B 2 below.

Lastly, we briefly consider the effect of field symmetry on the maximum compression, $\bar{\rho}_{\max }$, of the inner fluid, which we define as the volume-average density of the inner fluid at the time of near stagnation of the inner fluid, that is, at the time just after first reshock of the DI. Since effect of field symmetry is also of interest, we also define the standard deviation, $s(\rho)$, of the inner fluid density at this time, for each configuration. Table II shows estimates for these for the field configurations considered here, and suggests that the configuration of the magnetic field does not appreciably affect the maximum compression of the inner fluid. These values are approximate and depend on the time of measurement, called $t_{R}$. 


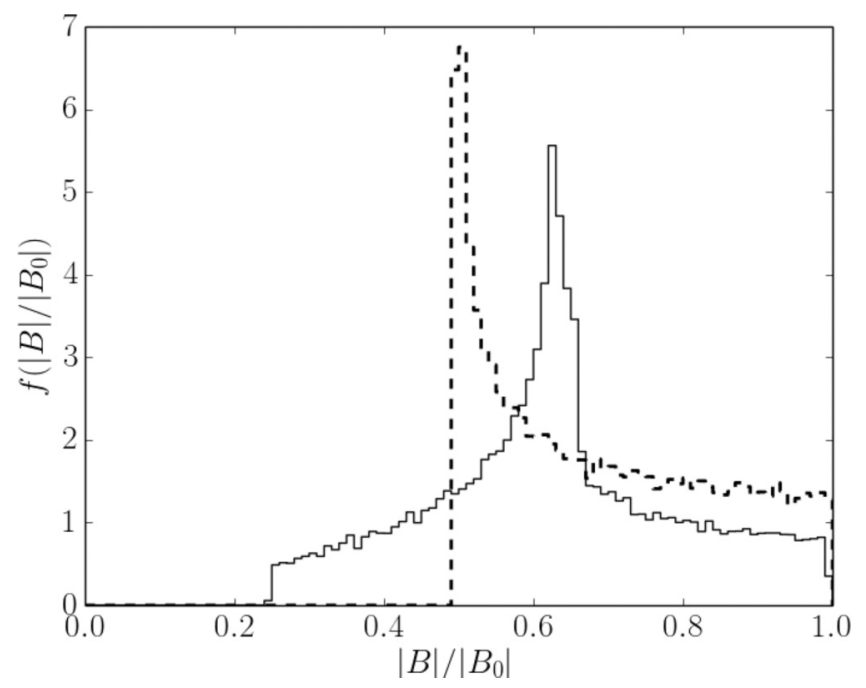

FIG. 9. Probability density functions (PDFs) showing strength distribution across the unit sphere for configurations 2 (dashed) and 6 (solid). Configuration 1, which is not shown, has a delta-function distribution at $|B| /\left|B_{0}\right|=1$ since it is uniform. Evidently, configuration 6 tends to have a higher field strength on the interface than configuration 2 for the given $\beta_{0 I}(=32)$, which partly explains the increased reduction in RM growth in case L6 over L2 seen in Fig. 7.

\section{Effect of field strength}

We lastly examine the influence of field strength on the degree of suppression, comparing the uniform and octahedral configurations at $\beta_{0 I}=8,16,32$. The two-loop configuration is not addressed here, since it is already known from Mostert et al. [20] that RM suppression in the two-loop field decreases relative to the uniform configuration with increasing field strength.

Figure 10 shows the rms amplitude growth for the different field strengths, comparing uniform and octahedral cases. It shows that, as expected, increasing field strength leads to an increased degree of suppression. In cases L6-8, L6-16, and L6-32 (L1-8, L1-16, L1-32) the perturbation amplitudes are reduced to approximately $50 \%, 68 \%$, and $76 \%(51 \%, 61 \%, 70 \%)$, respectively, of the unmagnetized case LO at the arbitrarily chosen representative time $t=0.5$. In particular, the peak perturbation amplitudes decrease with increasing field strength for both configurations. Note that, particularly for the strong-field case, the slow shock interacts with the DI in the octahedral field configuration, as seen in Fig. 11. The reason that this interference occurs in the octahedral case to a greater extent than in the uniform case is the greater slow magnetosonic speed near the initial Riemann interface at early times in the former case, leading to greater slow shock speeds and earlier slow shock-interface interaction. As the field strength increases, these slow shocks also become more compressive so that they disturb the gross symmetry of the DI if and when they interact with it.

TABLE II. Approximate mean density of reshocked inner fluid $\bar{\rho}_{\max }$ ("maximum compression") and standard deviation, $s(\rho)$, for tested field configurations, with time $t_{R}$ of measurement just after reshock of the DI.

\begin{tabular}{lcccc}
\hline \hline Configuration & L0 & L1-32 & L2-32 & L6-32 \\
\hline $\bar{\rho}_{\max }$ & 24.0 & 24.7 & 25.7 & 22.7 \\
$s(\rho)$ & 4.3 & 3.8 & 3.8 & 3.6 \\
$t_{R}$ & 0.87 & 0.86 & 0.86 & 0.87 \\
\hline \hline
\end{tabular}




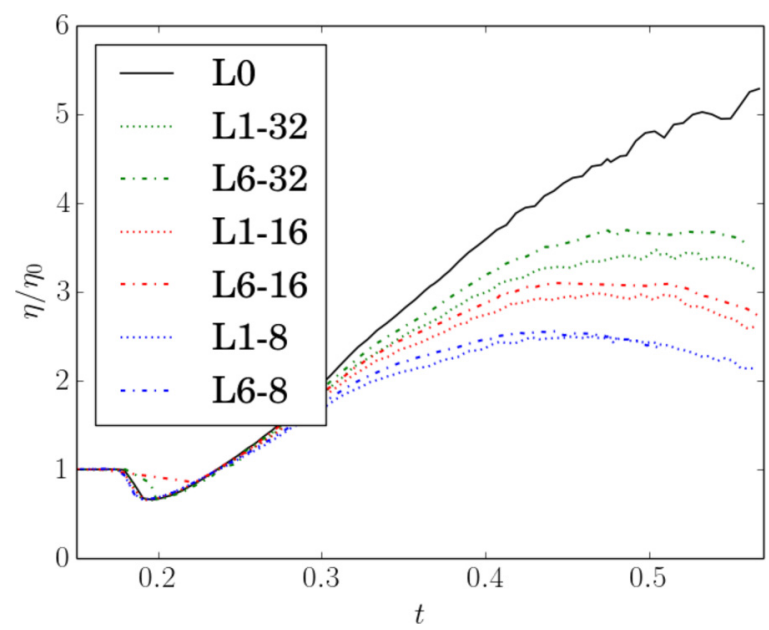

FIG. 10. Root-mean-square perturbation amplitude growth comparing uniform and octahedral cases across all field strengths. See Table I for description of abbreviations in the legend. Degree of RM suppression increases with field strength for both configurations. At the highest strength $\beta_{0 I}=8$, the relative suppression of the octahedral case (L6-8) versus the uniform (L1-8) is reduced over that of the others, probably owing to influence of the slow shock system.

However, the greater influence of the slow shock system in the octahedral case does not necessarily disturb the ability of the octahedral field to suppress the instability in general, as shown by Fig. 10 and suggested qualitatively by comparison of the peak amplitudes on the $x$ axis in Fig. 11. Furthermore, interaction of the slow shock with the DI may not occur in all cases anyway, for two reasons. First, whether or not the slow shocks reach the DI at all is largely problem dependent, since choosing a greater initial radius for the Riemann interface may ensure that the slow shocks remain far from the DI at late times. Second, one might choose a method of initialization not involving a Riemann problem of this kind: for example, the accelerating fast shock could in principle be initialized in the absence of other compressible waves by specifying the appropriate jump conditions $a b$ initio. We

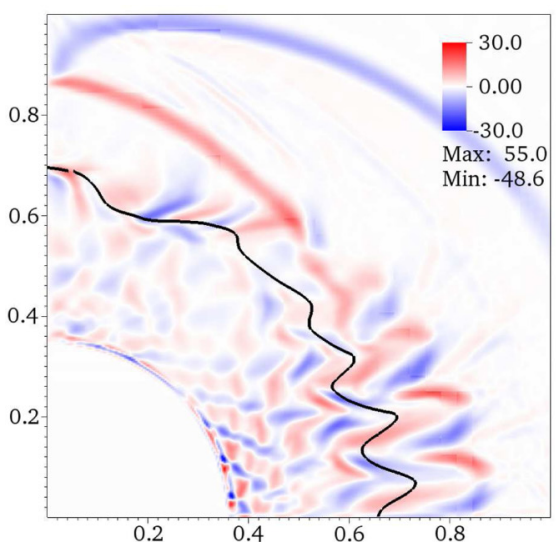

(a)

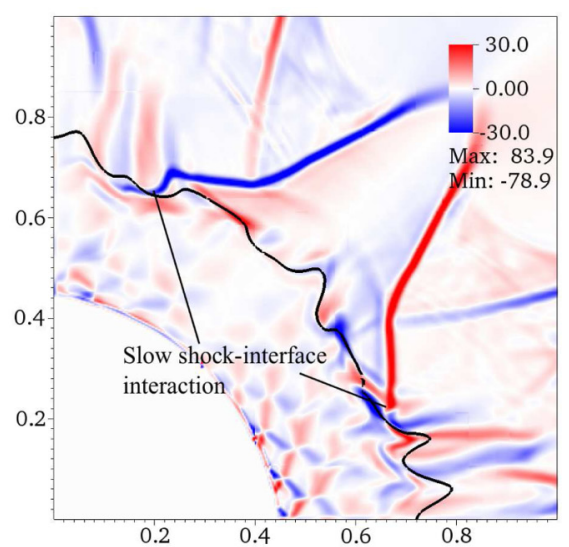

(b)

FIG. 11. $z$ vorticity in the $x-y$ plane for cases L1-8 and L6-8 at comparable times (see Table I) with the DI shown in black contour. The slow shock system visibly interferes with the DI in L6-8. Such interference is absent or very nearly so in L1-8. (a) $\mathrm{L} 1-8, t=0.507$ and (b) $\mathrm{L} 6-8, t=0.487$. 


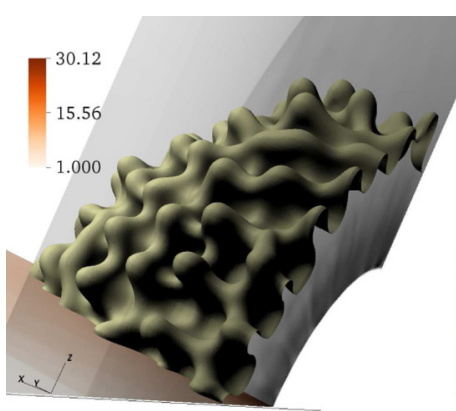

(a)

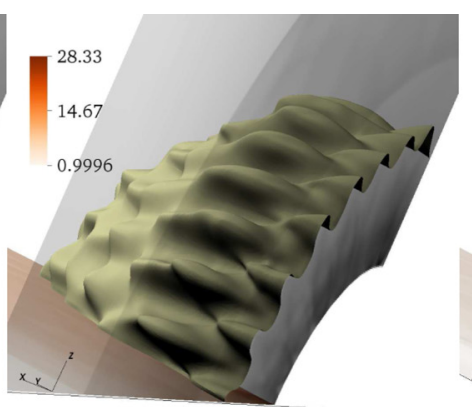

(b)

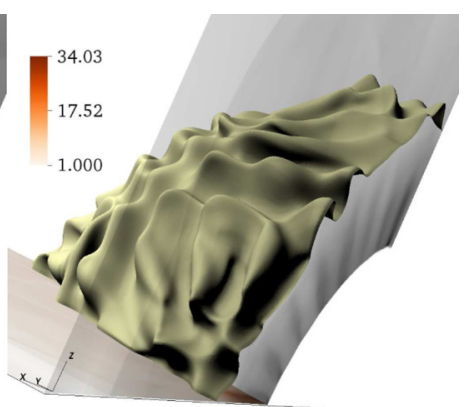

(c)

FIG. 12. Density isosurface and pressure contours for cases L0, L1-8, L6-8 (see Table I) at comparable times. The near plane is $y-z$. Qualitatively, the magnetized cases see reduction of perturbation growth due to RM instability. However, L1-8 visibly shows directional preference for RM suppression near the $y-z$ plane, while L6- 8 shows a more homogeneous suppression of RM instability across the isosurface. (a) L0, $t=0.510$, (b) $\mathrm{L} 1-8, t=0.507$, and (c) L6 - 8, $t=0.505$.

thus consider the slow shock system interference with the DI in this study as a potentially avoidable side effect.

Apart from the effect of the slow shock system on the DI in the octahedral case, the effect of the field on the extent of RM suppression in these problems is a matter of degree. In this sense, all fields are at least "critically strong" according to the criterion of Sano et al. [25], in the qualitative sense that the RM instability is suppressed. This consideration and more quantitative assessments are however complicated by, first, the three-dimensional, converging geometry of the problem considered; second, the great variation in field orientations and strength across the DI; third, the influence of Rayleigh-Taylor instability, particularly at late times; and fourth, the complex vorticity transport patterns present in the flow.

Finally, the effects of field symmetry on directional dependence of RM suppression are apparent particularly at the highest field strengths. Figure 12 shows density isosurfaces for cases L1-8 and L6-8 compared with L0. While the RM instability is clearly suppressed in both magnetized cases shown, L1-8 shows a clear directional preference in suppression in the $y-z$ plane, where the field is everywhere tangential to the interface (normal to the plane). The DI is corrugated nearly entirely within the $y-z$ plane, while perturbations in a direction normal to that plane are less evident. This is because the out-of-plane baroclinic vorticity transport does not affect the in-plane perturbations. Contrast this with L6-8, which owing to its symmetry shows a more homogeneous directional dependence. Effect of the slow shock system on the DI is not visible in Fig. 12.

\section{CONCLUDING REMARKS}

This study examines the effectiveness of an octahedrally symmetric magnetic field configuration in preserving the symmetry of an imploding flow in ideal magnetohydrodynamics and in the suppression of the Richtmyer-Meshkov instability in such flows. The field was chosen for its especially high degree of symmetry, and compared with other previously considered field configurations.

With the applied magnetic field, the imploding flow has a symmetry which matches that of the field, and exhibits less asymmetry in its imploding fast shock than the other field candidates, suggesting a more symmetric base flow. Further, the octahedrally symmetric field performs comparably to the uniform-field case and better than the two-loop field case in suppression of the Richtmyer-Meshkov instability in the global root-mean-square of perturbation amplitude growth.

This investigation highlights the potential usefulness of applied magnetic fields with high degrees of symmetry in converging flows where maintenance of implosion symmetry is a key performance 
factor, and that such fields can remain effective in suppression of hydrodynamic instabilities in flows of this kind.

\section{ACKNOWLEDGMENTS}

This research was supported by the KAUST Office of Sponsored Research under Award No. URF/1/2162-01. V.W. acknowledges support from an Australian Research Council Discovery Early Career Researcher Award (Project No. DE120102942).

[1] J. Lindl, O. Landen, J. Edwards, E. Moses, and NIC Team, Review of the National Ignition Campaign 2009-2012, Phys. Plasmas 21, 020501 (2014).

[2] R. D. Richtmyer, Taylor instability in shock acceleration of compressible fluids, Commun. Pure Appl. Math. 13, 297 (1960).

[3] E. E. Meshkov, Instability of the interface of two gases accelerated by a shock wave, Fluid Dyn. 4, 101 (1969).

[4] G. H. Markstein, Flow disturbances induced near a slightly wavy contact surface, or flame front, traversed by a shock wave, J. Aero. Sci. 24, 238 (1957).

[5] L. Rayleigh, Scientific Papers, Vol. II (Cambridge University Press, Cambridge, England, 1900).

[6] G. I. Taylor, The instability of liquid surfaces when accelerated in a direction perpendicular to their planes, i, Proc. R. Soc. London A 201, 192 (1950).

[7] A. B. Sefkow, S. A. Slutz, J. M. Koning, M. M. Marinak, K. J. Peterson, D. B. Sinars, and R. A. Vesey, Design of magnetized liner inertial fusion experiments using the $\mathrm{Z}$ facility, Phys. Plasmas (1994-present) 21, 072711 (2014).

[8] M. Hohenberger, P.-Y. Chang, G. Fiksel, J. P. Knauer, R. Betti, F. J. Marshall, D. D. Meyerhofer, F. H. Séguin, and R. D. Petrasso, Inertial confinement fusion implosions with imposed magnetic field compression using the OMEGA laser, Phys. Plasmas 19, 056306 (2012).

[9] P. Y. Chang, G. Fiksel, M. Hohenberger, J. P. Knauer, R. Betti, F. J. Marshall, D. D. Meyerhofer, F. H. Séguin, and R. D. Petrasso, Fusion Yield Enhancement in Magnetized Laser-Driven Implosions, Phys. Rev. Lett. 107, 035006 (2011).

[10] L. J. Perkins, B. G. Logan, G. B. Zimmerman, and C. J. Werner, Two-dimensional simulations of thermonuclear burn in ignition-scale inertial confinement fusion targets under compressed axial magnetic fields, Phys. Plasmas (1994-present) 20, 072708 (2013).

[11] R. Samtaney, Suppression of the Richtmyer-Meshkov instability in the presence of a magnetic field, Phys. Fluids 15, L53 (2003).

[12] V. Wheatley, R. Samtaney, and D. I. Pullin, The Richtmyer-Meshkov instability in magnetohydrodynamics, Phys. Fluids 21, 082102 (2009).

[13] V. Wheatley, D. I. Pullin, and R. Samtaney, Regular shock refraction at an oblique planar density interface in magnetohydrodynamics, J. Fluid Mech. 522, 179 (2005).

[14] V. Wheatley, D. I. Pullin, and R. Samtaney, Stability of an Impulsively Accelerated Density Interface in Magnetohydrodynamics, Phys. Rev. Lett. 95, 125002 (2005).

[15] V. Wheatley, R. Samtaney, D. I. Pullin, and R. M. Gehre, The transverse field Richtmyer-Meshkov instability in magnetohydrodynamics, Phys. Fluids 26, 016102 (2014).

[16] J. Cao, Z. Wu, H. Ren, and D. Li, Effects of shear flow and transverse magnetic field on Richtmyer-Meshkov instability, Phys. Plasmas 15, 042102 (2008).

[17] D. I. Pullin, W. Mostert, V. Wheatley, and R. Samtaney, Converging cylindrical shocks in ideal magnetohydrodynamics, Phys. Fluids (1994-present) 26, 097103 (2014).

[18] W. Mostert, V. Wheatley, R. Samtaney, and D. I. Pullin, Effects of seed magnetic fields on magnetohydrodynamic implosion structure and dynamics, Phys. Fluids (1994-present) 26, 126102 (2014).

[19] A. Bakhsh, S. Gao, R. Samtaney, and V. Wheatley, Linear simulations of the cylindrical RichtmyerMeshkov instability in magnetohydrodynamics, Phys. Fluids 28, 034106 (2016). 
[20] W. Mostert, V. Wheatley, R. Samtaney, and D. I. Pullin, Effects of magnetic fields on magnetohydrodynamic cylindrical and spherical Richtmyer-Meshkov instability, Phys. Fluids 27, 104102 (2015).

[21] J. P. Goedbloed, R. Keppens, and S. Poedts, Advanced Magnetohydrodynamics (Cambridge University Press, New York, 2010).

[22] M. Lombardini, D. I. Pullin, and D. I. Meiron, Turbulent mixing driven by spherical implosions. Part 1. Flow description and mixing-layer growth, J. Fluid Mech. 748, 85 (2014).

[23] W. R. Smythe, Static and Dynamic Electricity (McGraw-Hill Book Company, New York, 1950).

[24] B. C. Carlson, Computing elliptic integrals by duplication, Numer. Math. 33, 1 (1979).

[25] T. Sano, T. Inoue, and K. Nishihara, Critical Magnetic Field Strength for Suppression of the RichtmyerMeshkov Instability in Plasmas, Phys. Rev. Lett. 111, 205001 (2013).

[26] T. Sano, K. Nishihara, C. Matsuoka, and T. Inoue, Magnetic field amplification associated with the Richtmyer-Meshkov instability, Astrophys. J. 758, 126 (2012).

[27] G. Dimonte and P. Ramaprabhu, Simulations and model of the nonlinear Richtmyer-Meshkov instability, Phys. Fluids 22, 014104 (2010).

[28] R. Samtaney, P. Colella, T. J. Ligocki, D. F. Martin, and S. C. Jardin, An adaptive mesh semi-implicit conservative unsplit method for resistive MHD, J. Phys.: Conf. Ser. 16, 40 (2005).

[29] P. Colella, D. Graves, J. N. Johnson, H. S. Johansen, N. D. Keen, T. Ligocki, D. F. Martin, P. W. McCorquodale, D. Modiano, P. O. Schwartz, T. D. Sternberg, and B. Van Straalen, Chombo Software Package for AMR Applications Design Document, Lawrence Berkeley National Laboratory (Applied Numerical Algorithms Group, Berkeley, CA, 2012).

[30] V. Wheatley, R. M. Gehre, R. Samtaney, and D. I. Pullin, The magnetohydrodynamic Richtmyer-Meshkov instability: The oblique field case, in 29th International Symposium on Shock Waves (Springer, New York, 2013). 